\title{
Erratum to: Virtual labyrinth model of vestibular afferent excitation via implanted electrodes: validation and application to design of a multichannel vestibular prosthesis
}

\author{
Russell Hayden · Stacia Sawyer • Eric Frey • \\ Susumu Mori • Americo A. Migliaccio • \\ Charles C. Della Santina
}

Published online: 3 April 2011

(C) Springer-Verlag 2011

\section{Erratum to: Exp Brain Res}

DOI 10.1007/s00221-011-2599-x

In the original publication of this article, in Fig. 8e-h, all black-solid data traces should be black-single-short-dash (as for the Utricle in the legend), and all black-long-singledash data traces should be black-dash dot (as for the Saccule in the legend). All green solid lines should be green-dashed. Moreover, Fig. 9 was missing the word "Utricle" next to the single-dashed line in the legend. The corrected figures are presented in following page:

The online version of the original article can be found under doi:10.1007/s00221-011-2599-x.

R. Hayden $\cdot$ S. Sawyer $\cdot$ E. Frey $\cdot$ S. Mori $\cdot$

A. A. Migliaccio - C. C. Della Santina ( $\varangle)$

Johns Hopkins Vestibular NeuroEngineering Laboratory,

601 N. Caroline Street, Rm 6253, Baltimore,

MD 21287-0910, USA

e-mail: charley.dellasantina@jhu.edu

R. Hayden - A. A. Migliaccio - C. C. Della Santina

Departments of Otolaryngology-Head and Neck Surgery,

Johns Hopkins University School of Medicine,

Baltimore, MD, USA

R. Hayden - A. A. Migliaccio - C. C. Della Santina

Department of Biomedical Engineering,

Johns Hopkins University School of Medicine,

Baltimore, MD, USA

S. Sawyer $\cdot$ E. Frey $\cdot$ S. Mori

Department of Radiology,

Johns Hopkins University School of Medicine,

Baltimore, MD, USA 

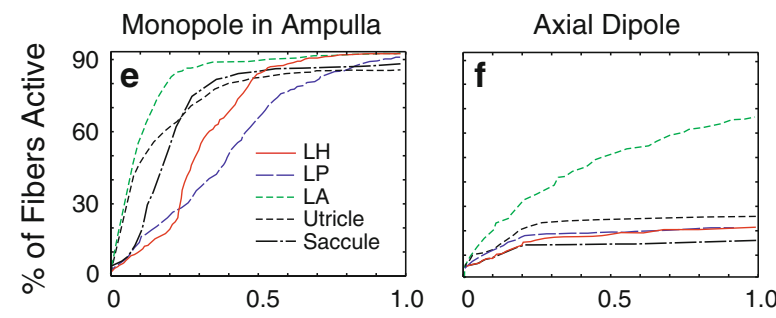

Transverse Parallel Dipole

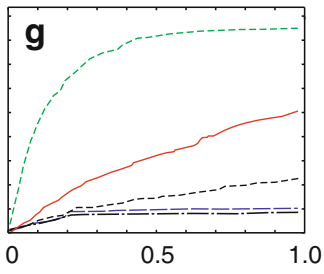

Transverse Perpendicular Dipole

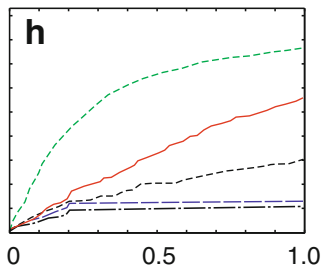

Stimulus Current (symmetric, cathodic-first biphasic pulse), mA

Fig. 8 e-h Simulated fiber recruitment plots for each anterior canal case. Transverse/parallel dipole outperforms other configurations with regard to selectivity

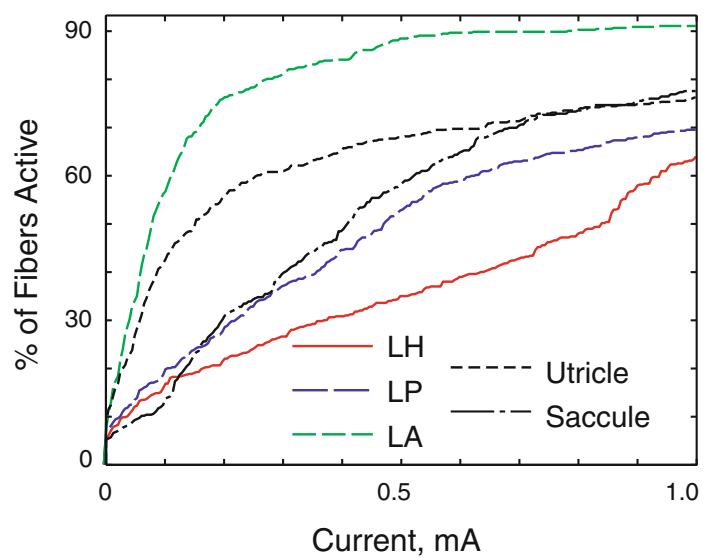

Fig. 9 Model recruitment curve for monopolar anterior canal electrode case shown in Fig. 8a but using cathodic-first pseudomonophasic stimuli. Improved selectivity is apparent compared to standard symmetric biphasic pulse stimulus (Fig. 8e) 